\title{
BMJ Open Effectiveness of a web-based decision aid for patients with generalised anxiety disorder: a protocol for a randomised controlled trial
}

\author{
Lilisbeth Perestelo-Pérez,, ${ }^{1,2}$ Amado Rivero-Santana, ${ }^{2,3}$ Vanesa Ramos-García (D) ,3,4 \\ Yolanda Álvarez-Pérez, ${ }^{3}$ Andrea Duarte-Díaz, ${ }^{3,4}$ Alezandra Torres-Castaño, ${ }^{3}$ \\ Maria del Mar Trujillo-Martín, ${ }^{2,3}$ Tasmania Del Pino-Sedeño, ${ }^{3}$ \\ Ana Isabel González-González (1) , 2,5,6 Pedro Serrano-Aguilar ${ }^{1,2}$
}

To cite: Perestelo-Pérez L, Rivero-Santana A, RamosGarcía V, et al. Effectiveness of a web-based decision aid for patients with generalised anxiety disorder: a protocol for a randomised controlled trial. BMJ Open 2020;10:e039956. doi:10.1136/ bmjopen-2020-039956

- Prepublication history and additional materials for this paper is available online. To view these files, please visit the journal online (http://dx.doi org/10.1136/bmjopen-2020039956).

Received 30 April 2020 Revised 03 November 2020 Accepted 11 November 2020

D) Check for updates

(c) Author(s) (or their employer(s)) 2020. Re-use permitted under CC BY-NC. No commercial re-use. See rights and permissions. Published by BMJ.

For numbered affiliations see end of article.

\section{Correspondence to} Dr Lilisbeth Perestelo-Pérez; lilisbeth.peresteloperez@sescs. es

\section{ABSTRACT}

Introduction Patients with generalised anxiety disorder (GAD) have concerns and needs about their health and the healthcare they receive. Patient decision aids (PtDAs) are tools that assist patients in making health decisions, when there is uncertainty about treatment choice, incorporating their personal preferences and values about the available treatment options. PtDAs can improve shared decision-making and lead to better treatment outcomes. The aim of this study is to evaluate the effectiveness of a web-based PtDA for patients with GAD in primary care (PC).

Methods and analysis The general study design is comprised of two stages: (1) development of a web-based PtDA for patients with GAD, derived from an evidence-based Clinical Practice Guideline and (2) assessment of the effectiveness of the PtDA in a randomised controlled trial (RCT) design, in PC centres in Tenerife (Spain). This RCT will be carried out with 124 patients with GAD, comparing the PtDA to a fact sheet with general information on mental health. Patients will review the PtDA in one session accompanied by a researcher. Post-intervention measures will be administered immediately after the intervention and at 3-month follow-up. The primary outcome will be decisional conflict. Secondary outcomes will include knowledge about GAD and its treatment, treatment preference, concordance between treatment preference and choice, and decision quality (knowledge $\geq 60 \%$ and concordant decision)

Ethics and dissemination The project received ethics approval from the local committee at Nuestra Señora de la Candelaria (HUNSC) University Hospital in Santa Cruz de Tenerife (code: CHUNSC_2019_58). Informed consent will be obtained from each participant before randomisation. Results from the trial will be submitted for publication in international peer-reviewed scientific journals and will be disseminated through workshops and local and international conferences. Trial registration number NCT04364958.

\section{INTRODUCTION}

Anxiety disorders (ADs) are some of the most prevalent mental health disorders, twice as common in women than men. ${ }^{2}$ They affect 61.5 million people in Europe, ${ }^{3}$ and findings from a recent prevalence study in
Strengths and limitations of this study

- This study will provide the first evidence on the effectiveness of a patient decision aid (PtDA) for patients with generalised anxiety disorder (GAD).

- The PtDA will be developed according to the International Patients Decision Aid Standards guidelines and based on the results of a Clinical Practice Guideline for GAD.

- The primary care centres randomly selected for this trial will provide a diverse and representative sample of patients with GAD.

- The application of the PtDA by a researcher reduces external validity, but the PtDA will be accessible to patients during the 3 months of follow-up.

- The impossibility of blinding participants and researchers and the self-reported nature of the outcome measures imply a risk of bias inherent to this type of study.

Spain indicated that about $11 \%$ of adults are affected by an $\mathrm{AD} .{ }^{4}$ ADs have an important negative impact on the health-related quality of life of patients as well as in their economies, due mostly to indirect costs. ${ }^{56}$

In primary care $(\mathrm{PC})$ settings, $\mathrm{ADs}$ are the most common mental disorders, ${ }^{78}$ and among them, the generalised anxiety disorder (GAD) is one of the most prevalent. ${ }^{8}$ However, due to the disorder's complexity and its frequent association with other conditions such as depression, its clinical recognition and management is becoming more difficult. ${ }^{9}$ GAD is characterised by persistent, excessive and uncontrollable anxiety and worry about everyday life events, and it is also frequently associated with interpersonal difficulties. ${ }^{10}$ This disorder displays periods of recurrence and remission, a variable course that can 
often result in functional and cognitive impairment and a reduction in health-related quality of life. ${ }^{11}$

Treatment options for GAD include pharmacotherapy (eg, selective serotonin reuptake inhibitors, selective serotonin-norepinephrine reuptake inhibitors, benzodiazepines) and psychological treatment (i.e., cognitive behavioural therapy, applied relaxation, ${ }^{11}$ metacognitive therapy, acceptance-based therapy or mindfulness). ${ }^{1213}$ As well as in other mental disorders, a stepped-care model, in which therapies are applied sequentially depending on the severity of the disorder and the response to previous treatments, is recommended. The stepped-care model is used to organise the provision of services and to help people with GAD, their families and healthcare professionals to choose the most effective interventions for each case. $^{11}$

Shared decision-making (SDM) is currently advocated as the gold standard of the interaction between patients and healthcare providers, both from an ethical (i.e., respecting patient's autonomy) and a practical perspective. SDM is aimed at incorporating patients' preferences and values regarding available treatment options into the decision-making process, avoiding professionals' paternalism in their relationship and communication with patients. SDM should take place in all phases of care to facilitate evidence-based information about treatments and discussion of the possible options. ${ }^{14}{ }^{15}$ The final aim of SDM is to help patients make decisions that are objectively informed and congruent with their own values regarding the treatments' potential consequences. ${ }^{16}$

Patient decision aids (PtDAs) are one of the most used interventions to promote SDM between healthcare professionals and patients. These tools can be developed in different formats (e.g., booklet, video, software, web) and include evidence-based information about the target disease and the available treatment options, with quantitative empirical data about potential benefits and risks of complications/adverse effects. They also try to promote an explicit or implicit reflection in patients about their own values and preferences concerning such potential consequences of treatments. ${ }^{17}$ PtDAs can be used before the clinical encounter, preparing patients to discuss their concerns and preferences about the options or in interaction with the professional during the consultation. The last update of the Cochrane systematic review of PtDAs for treatment or diagnostic decisions ${ }^{15}$ showed, compared with control groups, a pooled $23 \%$ relative improvement in objective knowledge about the disorder and treatments and twice as many patients with accurate risk perceptions and decisions congruent with their own values, respectively. PtDAs also decrease decisional conflict (i.e., uncertainty about own knowledge and preferences, and about the course of action to take) and reduce the number of people who are passive in decision-making by $32 \% .^{15}$

Nevertheless, in the field of mental health and especially in GAD, the number of interventions to promote
SDM is still limited. ${ }^{18-20}$ In the above mentioned Cochrane review, none of the 105 randomised trials addressed patients with ADs. Several studies have shown that many mental health patients want to be involved in the decisions about their care ${ }^{2122}$ and that clinical experts in specialised psychiatric care give value to interventions to promote SDM. ${ }^{23}{ }^{24}$ Therefore, the development and assessment of PtDAs in the field of ADs could help to reduce this gap and help patients to participate in the decision-making process.

In recent decades, working groups such as the Cochrane Collaboration have developed strategies that link the recommendations of Clinical Practice Guidelines (CPGs) and PtDAs in order to improve clinical practice. ${ }^{25}$ The conclusions of a recent methodological report support the need to develop PtDAs based on CPGs, with the aim of improving the quality of the SDM process. ${ }^{26}$ Currently, The Evaluation Unit of the Canary Islands Health Service (SESCS) is coordinating the update of a CPG for the treatment of patient with GAD in $\mathrm{PC}^{27}$ for the Clinical Practice Guidelines Programme in the National Health System (NHS) of Spain. This programme, launched in 2006, is coordinated by GuíaSalud (www.guiasalud.es), an NHS organisation in which the 17 Spanish Communities and the Ministry of Health created as an instrument to improve the quality of healthcare in the NHS.

Derived from this evidence-based CPG, a PtDA for patients with GAD will be developed here, given that the preferences and values of patients with GAD across the care process are not usually explored, ${ }^{18}$ and that they may experience difficulties deciding whether or not to use pharmacological medication, psychological therapy or both. Therefore, given the existing shortage of PtDAs for patients with GAD, the aims of this study are (1) to develop a PtDA for patients with GAD using internationally recommended procedures and (2) to assess the effectiveness of the PtDA in terms of improving the decisional process in patients with GAD.

\section{METHODS/ANALYSIS}

The protocol has been developed following the Standard Protocol Items: Recommendations for Interventional Trials (SPIRIT) 2013 statement $^{28}$ (see online supplemental additional file 1). Any future protocol modification will be registered in the ClinicalTrials. gov database and communicated to the Scientific and Ethics Committee of the HUNSC (Tenerife, Spain).

\section{Study design}

The study design is comprised of two stages: (1) developing a web-based PtDA for patients with GAD based on the recommendations of a CPG and (2) assessing the effectiveness of this PtDA in a RCT design.

Following the methodological process of Elwyn et al, ${ }^{29}$ the development of the web-based PtDA will consist of the following phases: 
Step 1: web-based PtDA from CPG recommendations ${ }^{27}$ (composed of five sub-phases)

1. Synthesis of the available evidence: the recommendations from the updated CPG for the treatment of patients with $\mathrm{GAD}^{27}$ will be used to develop the PtDA. Other existing PtDAs addressing patients with GAD will be also taken into consideration.

2. Creation of project management groups (PMGs): the PMG will be composed of an Expert Committee with six to eight participants from primary and specialised healthcare (i.e., family physicians, psychiatrists, nurses and psychologists) and four to five patients with GAD and their relatives and/or caregivers. Furthermore, a technical team (web designers and developers) and a scientific team (clinical experts and researchers) will be created. The group is at the heart of the development process and the people in this group have editorial control and make the final decisions on content specification, design and testing phases. The group will also undertake the early evaluation during prototype testing.

3. Development of the PtDA: the safety and effectiveness evidence of the different treatment options, based on the recommendations of the $\mathrm{CPG},{ }^{27}$ will be analysed and discussed by the scientific team to provide the main contents and a recommended structure. Presentation designs and technical issues will be discussed with the technical team. Finally, a first version of the PtDA will be developed according to the International Patients Decision Aid Standards (IPDAS) ${ }^{30}$

4. Content review: All members of the Expert Committee will assess the first version of the PtDA. Presentation and content issues will be reviewed and refined by means of an iterative process. This review process will be carried out through virtual or face-to-face meetings. As a result of this process, a second version of the PtDA will be developed.

5. Field testing: ten patients with GAD (with all degrees of severity) will test the PtDA, evaluating its acceptability in terms of content (clarity, quantity and usefulness of information), format issues, structure and navigability. The scientific team will collect qualitative and quantitative information from field testing. After modifying these aspects highlighted by patients in order to improve the PtDA, it will be ready to move to step 2 (effectiveness assessment).

Step 2: assessment of the PtDA effectiveness

\section{Design and setting}

A parallel, multicentre RCT will be carried out in PC centres in Tenerife (Canary Islands, Spain). Centres will be randomly selected among all the PC centres in Tenerife, stratified by urban/rural. Participants will be randomly allocated to intervention (web-based PtDA) or control group (web-based fact sheet with general information on mental health). The study is expected to last
9 months (from the start of recruitment to the complete finalisation of the data).

\section{Eligibility criteria}

\section{Inclusion criteria}

Adult ( $\geq 18$ years) with a confirmed diagnosis of GAD (ICD-10 ${ }^{31}$ or DSM-V $\mathrm{V}^{10}$ codes: $300.02 ; \mathrm{F} 41.1$ ) by a healthcare professional, with a score $\geq 8$ in the GAD- $7,{ }^{32}$ with ability to speak and read Spanish and who agree to participate and sign the informed consent. (online supplemental additional file 2).

\section{Exclusion criteria}

Patients with a primary diagnosis other than GAD, a score $<8$ in the GAD-7 and those with significant physical or mental disability that prevents them from completing the study activities or those participating in other trials related to GAD treatment or education will be excluded.

\section{Intervention}

PtDA group

The web-based PtDA (shown on the computer) will include information about GAD symptoms, diagnosis, causes, benefits and risks associated with psychological and pharmacological treatments for GAD (based on the CPG-GAD). It will also include a values clarification exercise, in which patients will have to rate the importance attributed to the different aspects of treatments (eg, mode of administration, benefits, risks, time to improvement, probability of relapse).

\section{Fact sheet group}

Patients in the control group will receive a web-based fact sheet (one page shown on the computer) with general information on mental health.

\section{Outcomes measures}

Primary outcome

The primary outcome will be the decisional conflict regarding the treatment for GAD, assessed with the Decisional Conflict Scale (DCS) ${ }^{33}$ immediately after reviewing the PtDA. The DCS evaluates the level of patient's subjective knowledge, perceived support and uncertainty when they are confronted with a medical decision. It includes 16 items and five subscales: feeling informed, clear values about benefits or risks, support, uncertainly and effective decision. Scores are transformed to a 0-100 scale, with higher scores indicating more conflict. In previous studies addressed to other health conditions, the authors have obtained good internal consistency values (Cronbach's alpha: 0.88-0.90). ${ }^{34-36}$

\section{Secondary outcomes}

- Knowledge about the disorder and treatment alternatives: due to the lack of published instruments to measure patients' objective knowledge about GAD and its treatments, a new scale will be developed with a maximum of ten items, based on the contents of the PtDA. Its content will include types of pharmacological 
and psychological treatments, adverse effects of drugs recommended for GAD, continuation pharmacotherapy or time to improve with psychotherapy.

- Treatment preference: assessed with one item with four response alternatives: (pharmacological treatment, psychological treatment, combined pharmacological and psychological treatment or unsure).

- Actual treatment choice: patients' actual treatment will be assessed at 3 months, with the same categories of treatment preference.

- Concordance between preferred (post-intervention) and chosen (3 months) treatment: assessed by a binary variable (concordant/non-concordant) derived from the congruence between preference and choice.

- Decision quality: assessed by a binary variable (yes/ no) defined as a combination of adequate knowledge ( $\geq 60 \%$ of correct responses) and a choice concordant with preferences.

\section{Adjustment variables}

- Sociodemographical variables including age, sex, educational level (no studies, primary education, secondary education, university or equivalent studies), current occupation (active, unemployed, household work, sick leave, pensioner, retired) and marital status (married/partner, single, separated/divorced, widowed).

- Generalised anxiety disorder-7 (GAD-7).$^{32}$ The questionnaire is a one-dimensional self-administered scale that assesses the presence of GAD symptoms, as listed in the DSM-IV. The total score may be categorised into four groups of severity: minimal (0-4), mild (5-9 points), moderate (10-14 points) and severe (14-20 points). ${ }^{37}$

- Hospital Anxiety Depression Scale (HADS) ${ }^{38}{ }^{39}$ : this is a 14-item questionnaire ${ }^{3940}$ composed of two subscales (HAD-A: anxiety and HAD-D: depression), of 7 items each, that are scored from 0 to 3 . For both (anxiety and depression), the authors recommend a threshold of 8 points to detect possible cases of anxiety and depression and a score greater than 11 to indicate cases probable of anxiety and depression.

- Patient Assessment Measure (PAM) ${ }^{41}{ }^{42}$ The questionnaire consists of 13 items that assess knowledge, skills and confidence of people for self-care, measured by a Likert 1-4 scale with a total score between 0 and 100 (higher scores indicate more activation).

- Control Preference Scale (CPS) ${ }^{43} 44$ to know patients' preferred roles in treatment decision. It is comprised of five cards with each of them representing a different role in treatment decision-making using a statement and a caricature. These roles range from the patient making the treatment decisions, through the patient making the decisions jointly with the physician, to the physician making the decisions. The CPS implicates patients in making a series of paired comparisons to provide their total preference order over the five web cards. The patient can score on: active (active-active or active-collaborative), collaborative (collaborativeactive or collaborative-passive) or passive (passivecollaborative or passive-passive).$^{44}$

- Previous treatment: pharmacological or psychological treatment used throughout the previous year (doses and sessions) and the services where the patient receives such treatments (public, private or both).

\section{Adverse events}

No adverse events associated with this intervention are expected to occur in any of the groups. However, after the completion of post-intervention measures, patients will be asked about any undesired psychological effect derived from their participation in the study, the application of the PtDA or the completion of questionnaires.

\section{Participant timeline}

Table 1 shows the details about schedule of enrolment, interventions and assessments. ${ }^{28}$ At baseline, in the telephone call to recruit patients, those who agree to participate will complete the primary outcome (DCS), the knowledge measure, treatment preference, GAD-7, PAM and HADS. Sociodemographical and clinical variables will also be assessed.

In the subsequent appointment in the health centre, patients will complete the CPS. They will then be allocated to the review of the PtDA/fact sheet, and after that the DCS, knowledge and preferred treatment will be assessed.

Three months after the intervention, patients will be phoned to complete the DCS, the knowledge scale and the GAD-7 again. The actual treatment choice will also be recorded. Table 1 on schedule of enrolment, interventions, and assessments (SPIRIT) checklist.

\section{Sample size}

Assuming type I and II errors of 0.05 and 0.20 , respectively, a total of 124 patients (62 patients per group) are needed in order to detect a medium effect size (Cohen's $d=0.5$ ) in the primary dependent variable (DCS). Assuming that $20 \%$ of patients are lost to follow-up, the final sample size increases to 156 patients (78 per group).

\section{Recruitment and procedure}

Patient's recruitment will take place in different PC centres, from urban and rural areas in Tenerife (Canary Islands, Spain). The research team will contact the directors of the health centres by telephone to arrange one meeting with all the health professionals. In this session (lasting approximately $45 \mathrm{~min}$ ), they will be informed by a researcher about the study objectives and the recruitment plan. Those interested in collaborating will sign the informed consent (see online supplemental additional file 2). These professionals will identify patients meeting eligibility criteria, from the electronic medical records, and phone them to ask for their verbal consent to provide their contact data to the research team. Patients who accept will be contacted by a researcher, who will provide the entire study information and apply the GAD-7 to 
Table 1 Schedule of enrolment, interventions and assessments (SPIRIT checklist)

\begin{tabular}{|c|c|c|c|c|c|}
\hline \multirow[b]{3}{*}{ Timepoint } & \multicolumn{5}{|c|}{ Study period } \\
\hline & \multicolumn{2}{|c|}{ Before randomisation } & \multirow{2}{*}{$\begin{array}{l}\text { Allocation } \\
0\end{array}$} & \multicolumn{2}{|c|}{ Post-allocation } \\
\hline & $\begin{array}{l}\text { Baseline } \\
\text { telephone }\end{array}$ & $\begin{array}{l}\text { Baseline On- } \\
\text { site }\end{array}$ & & $\begin{array}{l}\text { Post- } \\
\text { intervention }\end{array}$ & $\begin{array}{l}\text { Follow-up (3 } \\
\text { months) }\end{array}$ \\
\hline \multicolumn{6}{|l|}{ Enrolment: } \\
\hline Eligibility screen & $x$ & & & & \\
\hline Allocation & & & $\mathrm{x}$ & & \\
\hline \multicolumn{6}{|l|}{ Interventions: } \\
\hline Web-based PtDA & & & $X$ & & \\
\hline Web-based fact sheet & & & $\mathrm{X}$ & & \\
\hline DCS & $x$ & & & $x$ & $\mathrm{x}$ \\
\hline Knowledge & $X$ & & & $x$ & $x$ \\
\hline CPS & & $\mathrm{X}$ & & & \\
\hline PAM & $x$ & & & & \\
\hline HADS & $\mathrm{X}$ & & & & \\
\hline Treatment preference & $\mathrm{x}$ & & & $\mathrm{X}$ & \\
\hline Concordance between preference and choice & & & & & $\mathrm{X}$ \\
\hline Decision quality & & & & & $\mathrm{X}$ \\
\hline
\end{tabular}

CPS, Control Preference Scale; DCS, Decisional Conflict Scale; GAD-7, Generalised Anxiety Disorder-7; HADS, Hospital Anxiety and Depression Scale; PAM, Patient Activation Measure; PtDA, Patient Decisional Aid; SPIRIT, Standard Protocol Items: Recommendations for Interventional Trials.

confirm a probable GAD case (score $\geq 8$ ). Patients who agree to participate will complete the baseline assessment in the same phone call (or in a second one, if the assessment is too onerous for them), and an individual appointment of approximately $60 \mathrm{~min}$ at their PC reference centre will be arranged. At this meeting, patients will read the study information sheet, sign the informed consent and complete the CPS. The researcher will then open the sealed envelope with patient allocation. Those allocated to the intervention group will review the webbased PtDA, with the help of the researcher if necessary, and finally they will fill in the questionnaires assessing the outcome measures in the same web interface. Patients allocated to the control group will review a web-based fact sheet with general information on mental health, and then they will complete the same questionnaires. Three months later, participants will be contacted by phone and assessed again. During these 3 months, the PtDA will be accessible for patients in the intervention group (see figure 1).

\section{Allocation and blinding}

Computerised simple randomisation will be performed centrally by a researcher not involved in the study. Sealed opaque envelopes with each patient allocation will be prepared, to be opened only after the patient has signed the informed consent in the appointment where the PtDA or control intervention will be applied. The nature of the intervention makes it impossible to blind patients and researchers to group allocation. The investigator responsible for data analysis will be blinded to the intervention assignment.

\section{Data management}

All information gathered will be stored with an ID code number to maintain anonymity and stored in an electronic database management system located on a secure server with password-controlled access provided for research data collection, according to the General Registry for the Protection of Personal Data (RGPD UE 679/2016) and Spanish Organic Law 3/2018, of December 5, on the Protection of Personal Data and Guarantee of Digital Rights. Databases will be designed to avoid downloading inappropriate values for every variable. The Research Ethics Committees, the representatives of the health authority and the personnel authorised by the promoter may only access to check personal data, clinical study 


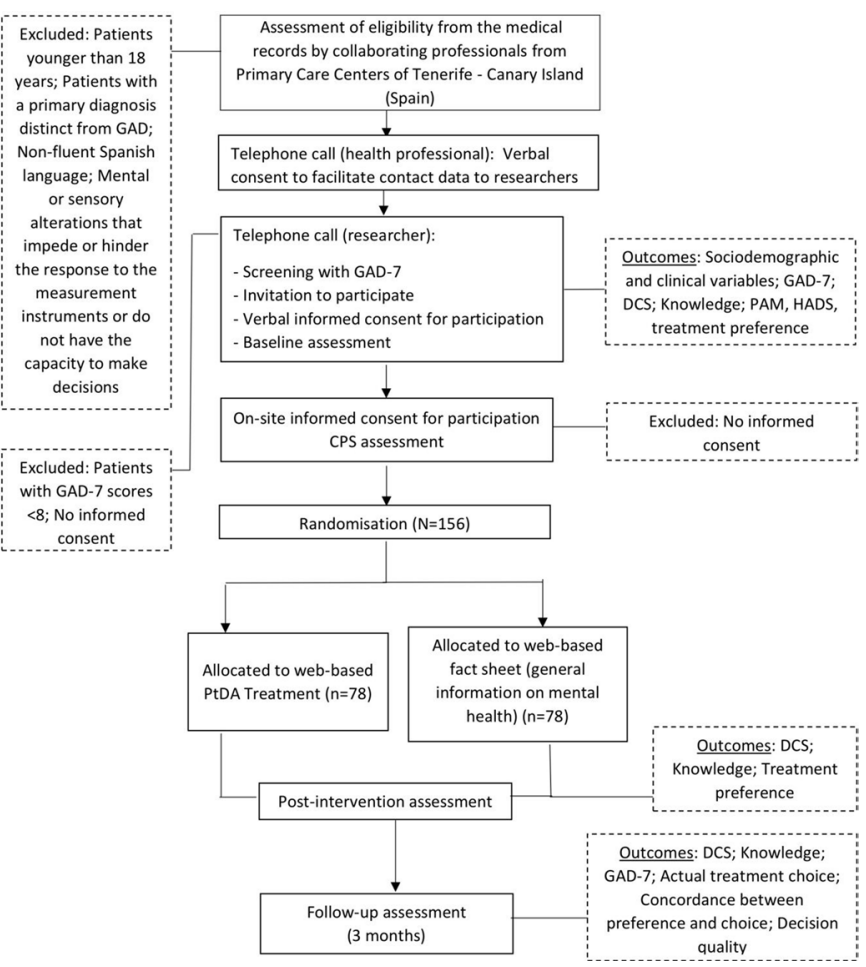

Figure 1 Flow of participants.

procedures and compliance with the rules of good clinical practice.

\section{Statistical analysis}

Descriptive statistics will be obtained for outcome and control variables. Analyses will be performed on an intention-to-treat basis, imputing missing values by means of multiple imputation (Markov Chain Monte Carlo method with 10 imputations per variable). The effect of the intervention on the primary outcome (DCS) and knowledge will be analysed by means of linear regression, adjusting for baseline scores, patient's activation (PAM) and other variables which show significant differences at baseline (assessed with Student's T-test and $\chi^{2}$ for continuous and categorical variables, respectively). The difference in the number of patients unsure about their treatment preference will be compared with the $\chi^{2}$ test.

Patients will be classified as concordant if their postintervention treatment preference coincides with their actual choice at 3 months (patients unsure at postintervention will be classified as non-concordant). The effect of the intervention in the number of concordant patients, as well as in those who made a quality decision (knowledge $\geq 60 \%$ and concordant choice) will be analysed by means of logistic regression, adjusting for the same covariates as those used for the continuous outcomes. A sensitivity analysis will be carried out excluding patients unsure at post-intervention. Analyses will be performed with the STATA.15 statistical programme.

\section{Patient and public involvement}

Neither patients nor public representatives were involved in the development of this study design but they will be involved in the development and validation of the PtDA.

\section{Challenges and limitations}

The main challenge for the successful development of the study is the collaboration of PC professionals in the recruitment of participants. In this regard, the research team has considerable experience conducting studies in PC, ${ }^{343645}$ and many of the potential participating centres have collaborated in these studies. Nonetheless, the current pandemic of COVID-19 could interfere or delay the recruitment or the application of the interventions in the PC centres, which could make an extension of the estimated duration of the study necessary. In case of extreme difficulty in applying the PtDA in-person, patients could review it online.

Another challenge is the potential overload of patients due to the various questionnaires applied; however, the selected instruments are relatively short, and the most onerous assessment is the baseline one, with approximately 60 items. If patients are overloaded, this assessment can be completed in a second telephone call. The application of the PtDA will be carried out by a researcher, thus avoiding problems related to professionals' time constraints.

The study has several limitations. Diagnosis of GAD will not be confirmed with a structured interview, but they will be screened with the GAD-7. We do not expect too many patients will be lost at follow-up, since its duration is 3 months and patients will be contacted by telephone. If they refuse to complete the follow-up questionnaires, we will try to record at least their actual treatment choice. Nonetheless, multiple imputation will be used to minimise the risk of attrition bias. At follow-up, health behaviours will not be assessed (e.g., adherence, physical activity), and although the GAD-7 questionnaire will be included in the assessment, there will be no hypothesising on changes on health outcomes. The primary aim of PtDAs is to promote involvement in decision-making and quality of decisions, and the evidence about their effects on health behaviours and outcomes is currently scarce and mixed.

\section{ETHICS AND DISSEMINATION}

The project received ethics approval from the local committees of the HUNSC in Santa Cruz de Tenerife (code: CHUNSC_2019_58). Informed consent will be obtained from each participant before randomisation. Results from the trial will be submitted for publication in international peer-reviewed scientific journals and will be disseminated through workshops and local and international conferences.

\section{TRIAL STATUS}

This study has been registered at ClinicalTrials.gov (identifier NCT04364958 number). The recruitment of 
patients will start in November 2020. The estimated end date of the recruitment will be August 2021.

\section{Author affiliations}

${ }^{1}$ Evaluation Unit (SESCS), Canary Islands Health Service (SCS), Tenerife, Spain

${ }^{2}$ Research Network on Health Services in Chronic Diseases (REDISSEC), Tenerife, Spain

${ }^{3}$ Canary Islands Health Research Institute Foundation, Tenerife, Spain

${ }^{4}$ University of La Laguna, Tenerife, Spain

${ }^{5}$ Goethe-Universitat Frankfurt am Main Institut fur Allgemeinmedizin, Frankfurt am Main, Germany

${ }^{6}$ Centro de Salud Vicente Muzas, Gerencia Asistencial de Atención Primaria, Servicio Madrileño de Salud, Madrid, Spain

Contributors LP-P, AR-S and VR-G designed the study and wrote the first draft of the protocol. YA-P, MMT-M, TDP-S contributed to the refinement of the study and reviewed and provided critical input to the study design and protocol. AT-C, AD-D, AR-S, LP-P, VR-G and YA-P will contribute to interpretation and dissemination. AD-D and AR-S will conduct statistical analyses. AIG-G and PS-A provided additional input about the methodology design. AD-D, AIG-G, AT-C, MMT-M, PS-A, TDP-S and YA-P contributed to critically revising the protocol manuscript and approved the final version.

Funding The authors have not declared a specific grant for this research from any funding agency in the public, commercial or not-for-profit sectors.

Competing interests None declared.

Patient consent for publication Not required.

Provenance and peer review Not commissioned; externally peer reviewed.

Supplemental material This content has been supplied by the author(s). It has not been vetted by BMJ Publishing Group Limited (BMJ) and may not have been peer-reviewed. Any opinions or recommendations discussed are solely those of the author(s) and are not endorsed by BMJ. BMJ disclaims all liability and responsibility arising from any reliance placed on the content. Where the content includes any translated material, BMJ does not warrant the accuracy and reliability of the translations (including but not limited to local regulations, clinical guidelines, terminology, drug names and drug dosages), and is not responsible for any error and/or omissions arising from translation and adaptation or otherwise.

Open access This is an open access article distributed in accordance with the Creative Commons Attribution Non Commercial (CC BY-NC 4.0) license, which permits others to distribute, remix, adapt, build upon this work non-commercially, and license their derivative works on different terms, provided the original work is properly cited, appropriate credit is given, any changes made indicated, and the use is non-commercial. See: http://creativecommons.org/licenses/by-nc/4.0/.

ORCID iDs

Vanesa Ramos-García http://orcid.org/0000-0002-9106-2420

Ana Isabel González-González http://orcid.org/0000-0002-1707-0596

\section{REFERENCES}

1 WHO. Mental health in the workplace [online]. World Health Organization, 2017. https://www.who.int/mental_health/es/

2 Stein DJ, Lim CCW, Roest AM, et al. The cross-national epidemiology of social anxiety disorder: data from the world mental health survey initiative. BMC Med 2017;15:143.

3 Begum M, Lewison G, Wölbert E, et al. Mental health disorders research in Europe, 2001-2018. Evid Based Ment Health 2020;23:15-20.

4 Villagrasa B, Olaya B, Lopez-Anton R, et al. Prevalence of anxiety disorder among older adults in Spain: a meta-analysis. $J$ Affect Disord 2019;246:408-17.

5 Rovira J, Albarracin G, Salvador L, et al. The cost of generalized anxiety disorder in primary care settings: results of the ANCORA study. Community Ment Health J 2012;48:372-83.

6 Alonso J, Lépine J-P, ESEMeD/MHEDEA 2000 Scientific Committee. Overview of key data from the European study of the epidemiology of mental disorders (ESEMeD). J Clin Psychiatry 2007;68:3-9.

7 Metzler DH, Mahoney D, Freedy JR. Anxiety disorders in primary care. Prim Care 2016;43:245-61.

8 Bados A. Trastorno de ansiedad generalizada [online], 2017. Available: http://hdl.handle.net/2445/115724
9 Parmentier H, García-Campayo J, Prieto R. Comprehensive review of generalized anxiety disorder in primary care in Europe. Curr Med Res Opin 2013;29:355-67.

10 American Psychiatric Association. Diagnostic and Stadistical manual of mental disorders: DSM-V. 5th edn. Washington, DC: American Psychiatric Association, 2013.

11 National Institute for Health and Care Excellence (NICE). Generalised anxiety disorder and panic disorder in adults: management [online], 2019. Available: https://www.nice.org.uk/guidance/cg113/chapter/ 1-Guidance\#stepped-care-for-people-with-gad [Accessed 3 May 2020].

12 Dahlin M, Andersson G, Magnusson K, et al. Internet-delivered acceptance-based behaviour therapy for generalized anxiety disorder: a randomized controlled trial. Behav Res Ther 2016;77:86-95.

13 Hoge EA, Bui E, Marques L, et al. Randomized controlled trial of mindfulness meditation for generalized anxiety disorder. J Clin Psychiatry 2013;74:786-92.

14 Durand M-A, Stiel M, Boivin J, et al. Where is the theory? evaluating the theoretical frameworks described in decision support technologies. Patient Educ Couns 2008;71:125-35.

15 Stacey D, Légaré F, Lewis $\mathrm{K}$, et al. Decision aids for people facing health treatment or screening decisions. Cochrane Database Syst Rev 2017;19.

16 Winn K, Ozanne E, Sepucha K. Measuring patient-centered care: an updated systematic review of how studies define and report concordance between patients' preferences and medical treatments. Patient Educ Couns 2015;98:811-21.

17 Coulter A, Kryworuchko J, Mullen PD, et al. Chapter A: using a systematic development process, in update of the International Patient Decision Aids Standards (IPDAS) Collaborations' Background Document, Llewellyn-Thomas HA, Volk RJ, editors, 2012: 1-16.

18 Hurtado MM, Villena A, Vega A, et al. 'I have anxiety, but I have values and preferences' Experiences of users with generalized anxiety disorder: a qualitative study. Int $J$ Ment Health Nurs 2020:inm. 12690 .

19 Metz MJ, Veerbeek MA, Elfeddali I, et al. [Shared decision making in mental health care; evaluation of the added value for patients and clinicians]. Tijdschr Psychiatr 2019;61:487-97.

20 Alguera-Lara V, Dowsey MM, Ride J, et al. Shared decision making in mental health: the importance for current clinical practice. Australas Psychiatry 2017;25:578-82.

21 Fisher A, Sharpe L, Anderson J, et al. Development and pilot of a decision-aid for patients with bipolar II disorder and their families making decisions about treatment options to prevent relapse. PLOS One 2018;13:e0200490.

22 Tlach L, Wüsten C, Daubmann A, et al. Information and decisionmaking needs among people with mental disorders: a systematic review of the literature. Health Expect 2015;18:1856-72.

23 Rodenburg-Vandenbussche S, Carlier I, van Vliet I, et al. Patients' and clinicians' perspectives on shared decision-making regarding treatment decisions for depression, anxiety disorders, and obsessive-compulsive disorder in specialized psychiatric care. J Eval Clin Pract 2020;26:645-58.

24 Samalin L, Genty J-B, Boyer L, et al. Shared decision-making: a systematic review focusing on mood disorders. Curr Psychiatry Rep 2018;20:23

25 Alonso-Coello P, Oxman AD, Moberg J, et al. Grade evidence to decision (ETD) frameworks: a systematic and transparent approach to making well informed healthcare choices. 2: clinical practice guidelines. BMJ 2016;353:i2089.

26 Perestelo Pérez L, Salcedo Fernández F, Toledo Chávarri A, et al. Desarrollo de herramientas de ayuda para la toma de decisiones compartida derivadas de las recomendaciones de las guías de práctica clínica. Ministerio de Sanidad, Servicios Sociales e Igualdad. Servicio de Evaluación del Servicio Canario de la Salud. Informes de Evaluación de Tecnologías Sanitarias 2017.

27 Grupo de Trabajo de la Guia de Práctica Clínica para el Manejo de Pacientes con Trastornos de Ansiedad en Atención Primaria. Guía de Práctica Clínica para el Manejo de Pacientes con Trastornos de Ansiedad en Atención Primaria. Madrid; Plan Nacional para el SNS del MSC. Unidad de Evaluación de Tecnologías Sanitarias. Agencia Laín Entralgo: Guías de Práctica Clínica en el SNS: UETS No 2006/10, 2008.

28 Chan A-W, Tetzlaff JM, Altman DG, et al. Spirit 2013 statement: defining standard protocol items for clinical trials. Ann Intern Med 2013;158:200.

29 Elwyn G, Kreuwel I, Durand MA, et al. How to develop web-based decision support interventions for patients: a process map. Patient Educ Couns 2011;82:260-5. 
30 Coulter A, Stilwell D, Kryworuchko J, et al. A systematic development process for patient decision aids. BMC Med Inform Decis Mak 2013;13:S2.

31 Word Health Organization. The ICD-10 classification of mental and behavioural disorders: clinical descriptions and diagnostic guidelines [online], 1992. Available: https://apps.who.int/iris/handle/10665/ 37958

32 García-Campayo J, Zamorano E, Ruiz MA, et al. Cultural adaptation into Spanish of the generalized anxiety disorder-7 (GAD-7) scale as a screening tool. Health Qual Life Outcomes 2010;8:8.

33 Urrutia M, Campos S, O'Connor A. [Validation of a Spanish version of the Decisional Conflict scale]. Rev Med Chil 2008;136:1439-47.

34 Perestelo-Perez L, Rivero-Santana A, Sanchez-Afonso JA, et al. Effectiveness of a decision aid for patients with depression: a randomized controlled trial. Health Expect 2017;20:1096-105

35 Perestelo-Pérez L, Rivero-Santana A, Boronat M, et al. Effect of the statin choice encounter decision aid in Spanish patients with type 2 diabetes: a randomized trial. Patient Educ Couns 2016;99:295-9.

36 Perestelo-Perez L, Rivero-Santana A, Torres-Castaño A, et al. Effectiveness of a decision aid for promoting colorectal cancer screening in Spain: a randomized trial. BMC Med Inform Decis Mak 2019;19:8.

37 Spitzer RL, Kroenke K, Williams JBW, et al. A brief measure for assessing generalized anxiety disorder. Arch Intern Med 2006;166:1092.
38 Quintana JM, Padierna A, Esteban C, et al. Evaluation of the psychometric characteristics of the Spanish version of the hospital anxiety and depression scale. Acta Psychiatr Scand 2003;107:216-21.

39 Herrero MJ, Blanch J, Peri JM, et al. A validation study of the hospital anxiety and depression scale (HADS) in a Spanish population. Gen Hosp Psychiatry 2003;25:277-83.

40 Terol-Cantero MC, Cabrera-Perona V, Martín-Aragón M. Revisión de estudios de la Escala de Ansiedad Y Depresión Hospitalaria (had) en muestras españolas. An Psicol 2015;31:494.

41 Skolasky RL, Green AF, Scharfstein D, et al. Psychometric properties of the patient activation measure among multimorbid older adults. Health Serv Res 2011;46:457-78.

42 Moreno-Chico C, González-de Paz L, Monforte-Royo C, et al. Adaptation to European Spanish and psychometric properties of the patient activation measure 13 in patients with chronic diseases. Fam Pract 2017;34:627-34.

43 Degner LF, Sloan JA, Venkatesh P. The control preferences scale. Can J Nurs Res 1997;29:21-43.

44 De Las Cuevas C, Peñate W. Validity of the control preferences scale in patients with emotional disorders. Patient Prefer Adherence 2016;10:2351-6.

45 Perestelo-Pérez L, Rivero-Santana A, Boronat M, et al. Effect of the statin choice encounter decision aid in Spanish patients with type 2 diabetes: a randomized trial. Patient Educ Couns 2016;99:295-9. 